\title{
Metacyclic 2-groups with the same conjugacy classes and their commutativity degree
}

\author{
K. Moradipour ${ }^{1}$, N. H. Sarmin ${ }^{2^{*}}$ and A. Erfanian ${ }^{3}$ \\ ${ }^{1,2}$ Department of Mathematical Sciences, Faculty of Science and Ibnu Sina Institute for Fundamental Science Studies, \\ Universiti Teknologi Malaysia, 81310 UTM Johor Bahru, Johor, Malaysia
${ }^{3}$ Department of Pure Mathematics and Center of Excellence in Analysis on Algebraic Structure, Faculty of \\ Mathematical Sciences, Ferdowsi University of Mashhad, Mashhad, Iran \\ 1'kayvan.mrp@gmail.com, ${ }^{2}$ nhs@utm.my, ${ }^{3}$ erfanian@utm.my
}

\begin{abstract}
In this paper the number of conjugacy classes of $G$ for some non-abelian finite groups is computed. Also, the commuting probability of $G$, denoted by $P_{r}(G)$, is obtained. In particular, the commutativity degree of $G$ both in the split and non-split case is described.
\end{abstract}

Keywords: Conjugacy class, Commutativity degree, Split group.

\section{Introduction}

The commutativity degree of a finite group $G$ is the probability that a randomly selected pairs of elements of the group commute. The concept of commutativity degree or probability of commuting pairs of a group was established by Erdos and Turan (1968). They have shown that for a finite group $G, P_{r}(G)>\frac{\log \log G}{|G|}$.

Let $G$ be a group of finite order $n$. The probability $P_{r}(G)$, that two elements selected at random from $G$ are commutative is

$$
P_{r}(G)=\frac{|\Psi|}{n^{2}}=\frac{\left|\left\{(x, y) \in G^{2} \mid x y=y x\right\}\right|}{n^{2}} .
$$

In order to count the elements of $\Psi$, we have for each $x \in G$ the number of elements of $\Psi$ of the form $(x, y)$ is $\left|C_{G}(x)\right|$, where $C_{G}(x)$ is the centralizer of $x$ in $G$. Hence, we have $|\Psi|=\sum\left|C_{G}(x)\right|$, where the sum extends over all $x \in G$. We recall that if $x$ and $y$ are conjugate elements of $G$, then $C_{G}(x)$ and $C_{G}(y)$ are conjugate subgroups. Moreover, the number of elements in the conjugacy classes of $x$ is $\left[G: C_{G}(x)\right]$. Hence, if $x_{1}, \ldots, x_{k}$ are representatives of the conjugacy classes in $G$, then $|\Psi|=\sum_{i=1}^{k}\left[G: C_{G}\left(x_{i}\right)\right]\left|C_{G}\left(x_{i}\right)\right|=k \cdot n$.

Thus

$P_{r}(G)=\frac{k(G)}{|G|}$

where $k(G)$ is the number of conjugacy classes of $G$ and $|G|$ is the order of $G$. This formula has been proved by Gustafson (1973) and the method of the proof was used by Erdos and Turan (1968). Also, it has shown (Gustafson, 1973) that if $G$ is any non-abelian group, then the upper bound for $\frac{k(G)}{|G|}$ is $\frac{5}{8}$ thus $P(G) \leq \frac{5}{8}$.

Equation (1.1) shows that finding the commutativity degree of a group is equivalent to finding the number of conjugacy classes of the group.

There are several papers on the conjugacy classes and commutativity degree of finite $p$-groups including Research article CIndian Society for Education and Environment (iSee)
(Sherman, 1979; Huppert, 1998; Jaikin-Zapirai, 2003; Erfanian, 2009; Kanti \& Kumar, 2010). Many authors achieved significant results on the lower and upper bound for $k(G)$. For instance, In 1979, Sherman proved that if $G$ is a finite nilpotent group of nilpotency class $m$, then $k(G)>m|G|^{\frac{1}{m}}-m+1$ and then $k(G)>\log _{2}|G|$. Later Huppert (1998) showed that $k(G)>\log n$ for any nilpotent group $G$ of order $n$.

Recently, a general formula for the exact number of conjugacy classes and commutativity degree of two generators metacyclic $p$-groups has been computed by Moradipour et al. (2012). It has been shown that if $G$ is a metacyclic $p$-group with the following presentation:

If $G$ is nilpotent of class two, then

$G \cong\left\langle a, b \mid a^{p^{\alpha}}=b^{p^{\beta}}=1,[a, b]=a^{p^{\alpha-\gamma}}\right\rangle$

where $\alpha, \beta, \gamma \in N, \alpha \geq 2 \gamma, \beta \geq \gamma \geq 1$;

If $p$ is odd and the class of $G$ is greater than 2, then

$G \cong\left\langle a, b \mid a^{p^{\alpha}}=b^{p^{\beta}}=1,[b, a]=a^{p^{\alpha-\gamma}}\right\rangle$

where

$\alpha, \beta, \gamma \in N, \gamma-1<\alpha<2 \gamma, \beta \geq \gamma$;

$G \cong\langle a, b| a^{p^{\alpha}}=1, \quad b^{p^{\beta}}=a^{p^{\alpha-\varepsilon}}$,

$$
\left.[b, a]=a^{p^{\alpha-\gamma}}\right\rangle \text {, }
$$

where

$\alpha, \beta, \gamma \in N, \gamma-1<\alpha<2 \gamma, \beta \geq \gamma, \alpha<\beta+\varepsilon$; then, the commutativity degree of group $G$ is equal to $\frac{1}{p^{\gamma}}+\frac{1}{p^{\gamma+1}}-\frac{1}{p^{2 \gamma+1}}$

A group $G$ is called metacyclic if it contains a normal cyclic subgroup whose quotient is also cyclic. As an example:

Let

$G=\left\langle a, b \mid a^{16}=b^{2}=1 ; a^{b}=a^{9}\right\rangle$.

Then $G$ is a non-abelian metacyclic 2-group. Furthermore, there is a normal cyclic subgroup $\mathbb{N}$, whose 
quotient group is cyclic, We show that $\left\langle a^{4} \mathrm{~b}\right\rangle$ is a normal subgroup of $G$. For this conjugating $a^{4} \mathrm{~b}$ by $a$ yields $a\left(a^{4} b\right) a^{-1}=a^{-4} b=\left(a^{4} b\right)^{3}$.

This shows that $G$ has cyclic subgroups whose quotient groups are cyclic.

In this paper we focus on some specific metacyclic 2groups. Our basic goal is to compute the number of conjugacy classes and then commutativity degree of the generalized quaternion groups, dihedral groups, semidihedral groups and quasi dihedral groups. The following presentation is the generalized quaternion group $Q_{2^{\alpha+1}}$. Theorem 1. 1 (Beuerle, 2005) Let $G$ be a metacyclic 2group. Then

$G \cong\left\langle a, b \mid a^{2^{\alpha}}=1, b^{2}=a^{2^{\alpha-1}},[b, a]=a^{-2}\right\rangle$,

where $\alpha \geq 3$.

Theorem 1.2 is a presentation of dihedral group $D_{2^{\alpha+1}}$.

Theorem 1.2 (Beuerle, 2005) If $G$ is a metacyclic group, then

$G \cong\left\langle a, b \mid a^{2^{\alpha}}=b^{2}=1,[b, a]=a^{-2}\right\rangle$,

where $\alpha \geq 3$ :

The following group is the semi-dihedral group $S D_{2^{\alpha+1}}$.

Theorem 1.3 (Beuerle, 2005) If $\mathrm{G}$ is a metacyclic 2group, then

$G \cong\left\langle a, b \mid a^{2^{\alpha}}=b^{2}=1,[b, a]=a^{2^{\alpha-1}-2}\right\rangle$,

where $\alpha \geq 3$.

The group $Q D_{2^{\alpha+1}}$, quasi-dihedral group has the following presentation.

Theorem 1.4 (Beuerle, 2005) If $\mathrm{G}$ is a metacyclic group, then

$G \cong\left\langle a, b \mid a^{2^{\alpha}}=b^{2}=1,[b, a]=a^{2^{\alpha-1}}\right\rangle$,

where $\alpha \geq 3$.

Some basic results

In this section we give some results which are useful to prove our theorems. We use the notation $[b, a]=$ $b a b^{-1} a^{-1}=a^{b} a^{-1}$ for the commutator of $b$ and $a$.

Lemma 2.1 (Moradipour et al., 2012) Let $\alpha, \beta, r$ and $\varepsilon$ be integers with $\alpha, \beta$ non-negative and let

$G \cong\left\langle a, b \mid a^{p^{\alpha}}=1, b^{p^{\beta}}=a^{p^{\alpha-\varepsilon}}, a^{b}=a^{r}\right\rangle$,

be a non-abelian p-group, where $r=p^{\alpha-\gamma} \pm 1$. If $a, b \in G$ with $a=x^{i} y^{j}$ and $b=x^{s} y^{t}$, then the following hold in $\mathrm{G}$ :

(1) $a b=x^{i+s r^{j}} y^{j+t}$,

(2) $a^{b}=x^{s\left(1-r^{j}\right)+i r^{t}} y^{j}$

(3) $[a, b]=x^{i\left(1-r^{t}\right)+s\left(r^{j}-1\right)}$.

Lemma 2.2 (King, 1973) Let G be a non-abelian 2-group of type

$G(2, \alpha, \beta, \varepsilon, \gamma)=\left\langle a, b \mid a^{2^{\alpha}}=1, b^{2^{\beta}}=a^{2^{\alpha-\varepsilon}}, a^{b}=a^{r}\right\rangle$, where $r=2^{\alpha-\gamma}+1$. Then
(1) $|G|=2^{\alpha+\beta}$

(2) $Z(G)=\left\langle a^{2^{\gamma}}, b^{2^{\gamma}}\right\rangle$;

(3) $|Z(G)|=2^{\alpha+\beta-2 \gamma}$.

Lemma 2.3 (King, 1973) Let $\mathrm{G}$ be a metacyclic 2-group of type

$G(2, \alpha, \beta, \varepsilon, \gamma)=\left\langle a, b \mid a^{2^{\alpha}}=1, b^{2^{\beta}}=a^{2^{\alpha-\varepsilon}}, a^{b}=a^{t}\right\rangle$, where $r=2^{\alpha-\gamma}-1$. Then

(1) $|G|=2^{\alpha+\beta}$;

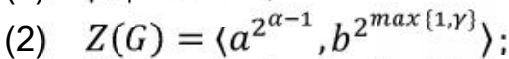

(3) $|Z(G)|=2^{\beta-\max \{1, \gamma\}+1}$.

If $G$ is a non-abelian $p$-group of order $p^{\alpha+\beta}$ with relation $b a=a^{r} b$, then each element in the group can be written in the unique form $a^{s} b^{t}$, where all possible value for $s$ and $t$ is $0 \leq s<p^{\alpha}, 0<t<p^{\beta}$. Now we are ready to find the conjugacy classes and commutativity degree of our groups.

Main theorems

Four theorems on $k(G)$ and $P_{r}(G)$ in terms of $\alpha$ are given as follows

Theorem 3.1 Let $\mathrm{G}$ be a non-abelian group. If

$G \cong\left\langle a, b \mid a^{2^{\alpha}}=b^{2}=1,[b, a]=a^{2^{\alpha-1}-2}\right\rangle$.

where $\alpha \geq 3$, then $k(G)=2^{\alpha-1}+3$.

Proof. This group is generalized quaternion group $Q_{2} \alpha+1$. By Lemma 2.3 the order of $G$ is $2^{\alpha+1}$ and $Z(G)=$ $\left\langle a^{2^{\alpha-1}}, b^{2}\right\rangle$ and $|Z(G)|=2$. We can write an arbitrary element of $G$ in the unique form $a^{i}$ and $a^{i} b$ where $1 \leq i \leq 2^{\alpha}-1$. From relation $[b, a]=a^{-2}$, we have $b a=a^{-1} b$ so any element of the form $b^{j} a^{i}=a^{i} b^{j}$ if $j$ is even and $b^{j} a^{i}=a^{-i} b^{j}$ if $j$ is odd. First we consider an element of the form $a^{i} \in G$ to conjugate by element $a^{t}$

$\left(a^{i}\right)^{a^{t}}=a^{t} a^{i} a^{-t}=a^{i}$

and

$\left(a^{i}\right)^{a^{t} b}=a^{t} b a^{i} b a^{-t}=a^{t} a^{-(t+i)} b=a^{-i}$.

Thus

$\left.a^{i}\right]=\left\{a^{i}, a^{-i}\right\}$.

In this case we have $2^{\alpha}-2$ non-central elements which are partitioned into 2 element conjugacy classes of the form $\left[a^{i}\right]$. Consequently we have $\left(2^{\alpha}-2\right)^{\prime} / 2=2^{\alpha-1}-$ 1 such classes. Next conjugate an element of the second form $a^{i} b$ by $a^{t}$ and $a^{t} b$ then

$\left(a^{i} b\right)^{a^{t}}=a^{t} a^{i} b a^{t}=a^{t+i} a^{t} b=a^{2 t+i} b$

and

$\left(a^{i} b\right)^{a^{t} b}=a^{t} b a^{i-t}=a^{t} a^{t-i} b=a^{2 t-i} b$.

Thus

$\left.a^{i} b\right]=\left\{a^{2 t+i} b, a^{2 t-i} b \mid 0 \leq t \leq 2^{\alpha}-1\right\}$.

Consequently,

$[a b]=\left\{a b, a^{3} b, \ldots, a^{2^{\alpha}-1} b\right\}=\left\{a^{i} b \mid i\right.$ is odd $\}$ 
and

$\left[a^{2} b\right]=\left\{b, a^{2} b, \ldots, a^{2^{\alpha}-2} b\right\}=\left\{a^{i} b \mid i\right.$ is even $\}$,

are 2 conjugacy classes including of all elements of the form $a^{i} b$ with $1 \leq i<2^{\alpha}$. Thus all of non-central elements of $G$ are consisted in one of the classes mentioned above. In addition,

$Z(G)=\left\langle a^{2^{\alpha-1}}, b^{2}\right\rangle=\left\langle a^{2^{\alpha-1}}\right\rangle$

and $|Z(G)|=2$. Therefore, the number of conjugacy classes of $G$ is equal to $2^{\alpha-1}+3$. Theorem 3.2 Let $\mathrm{G}$ be a non-abelian presented by

$G \cong\left\langle a, b \mid a^{2^{\alpha}}=b^{2}=1,[b, a]=a^{-2}\right\rangle$,

where $\alpha \geq 3$. Then $k(G)=2^{\alpha-1}+3$.

Proof. This group is the dihedral group $D_{2}{ }^{a+1}$ of order $2^{\alpha+1}$. The group $G$ is split extension and by Lemma 2.3 , $Z(G)=\left\langle a^{2^{a-1}}\right\rangle$ and $|Z(G)|=2$. From the relation $[b, a]=a^{-2}$, we have $b a=a^{-1} b$. We can write $G=\left\{a^{i} \mid 0 \leq i \leq 2^{\alpha}-1\right\} \cup\left\{a^{i} b \mid 0 \leq i \leq 2^{\alpha}-1\right\}$.

We now conjugate elements of $G$ by $a^{i}$ and $a^{i} b$ to find the conjugacy classes. Thus any element of the form $b^{j} a^{i}$ can be written by $b^{j} a^{i}=a^{i(-1)^{j}} b^{j}$. Suppose $0 \leq t \leq 2^{\alpha}-1$ then

$\left(a^{t}\right)^{a^{i}}:=a^{i} a^{t} a^{-i}=a^{t}$

and

$\left(a^{t}\right)^{a^{i} b}=a^{i} b a^{t} b a^{-i}=a^{i} b a^{t+i} b=a^{-t} b^{2}=a^{-t}$.

Thus

$\left[a^{t}\right]=\left\{a^{t}, a^{-t}\right\}$.

We suppose that $t \neq 2^{\alpha-1}, 0$. Then $2^{\alpha}-2$ non-central elements are partitioned into two element conjugacy classes of the form $\left[a^{t}\right]=\langle a\rangle$. Hence we have $\left(2^{\alpha}-\right.$ 2) $/ 2=2^{\alpha-1}-1$ such classes. Next we conjugate $b$ by $a^{-j}$ and $a^{j} b, \quad$ then

and

$(b)^{a^{j} b}=a^{j} b a^{-j}=a^{2 j} b$.

Hence

$[b]=\left[a^{2 j} b\right]=\left\{a^{2 j} b \mid 0 \leq j \leq \frac{2^{a}-1}{2}\right\}$

Here half of the elements of the form $a^{i} b$ are included by [b]. We should find ail further conjugacy classes including elements of the form $a^{i} b$ establishing with $[a b]$. Suppose $0 \leq j \leq 2^{\alpha}-1$ then

$(a b)^{a^{-j}}=a^{-j} a b a^{j}=a^{1-2 j} b$,

and

$(a b)^{a^{-j} b}=a^{-j} b a b^{2} a^{-j}=a^{2 j-1} b$.

Since

$\left\langle a^{2 j+1}\right\rangle=\left\{a^{i}: 0 \leq i<2^{\alpha}\right.$ and $i$ is odd $\}$,

then

$[a b]=\left[a^{2 j+1} b\right]=\left\{a^{i} b: 0 \leq i<2^{\alpha}\right.$ and $i$ is odd $\}$.
Hence, $[a b]$ includes the other half of the elements of the form $a^{i} b$. Therefore all classes containing elements of the form $a^{i} b$ with $0 \leq i \leq 2^{\alpha}-1$ have been found. Hence we have 2 conjugacy classes of the form $[a b]$ and $[a]$. All non-central elements of $G$ are included in one of the classes expressed above. As mentioned before we have $Z(G)=\left\langle a^{2^{\alpha-1}}, b^{2}\right\rangle=\left\langle a^{2^{\alpha-1}}\right\rangle$.

So $Z(G)$ contains $|Z(G)|=2$ conjugacy classes. Therefore we have

$k(G)=2+2^{\alpha-1}+1=2^{\alpha-1}+3$.

Theorem 3.3 Let $\mathrm{G}$ be a non-abelian 2-group. If

$G \cong\left\langle a, b \mid a^{2^{\alpha}}=b^{2}=1,[b, a]=a^{2^{\alpha-1}-2}\right\rangle$,

where $\alpha \geq 3$, then $k(G)=2^{\alpha-1}+3$.

Proof. This group is semi-dihedral group $S D_{2^{\alpha+1}}$ with order $2^{\alpha+1}$. Also,

$Z(G)=\left\langle a^{2^{\alpha-1}}, b^{2}\right\rangle=\left\langle a^{2^{\alpha-1}}\right\rangle$

and $|Z(G)|=2$. Each element of the group $G$ can be written in the unique form $a^{s}$ or $a^{s} b$ with $0 \leq s<2^{\alpha}$. We rewrite $[b, a]=a^{2^{\alpha-1}-2}$ to $b a=a^{r} b$ where $r=2^{\alpha-1}$ 1. we now conjugate elements of $G$ to find conjugacy classes. Suppose $a^{i} \in G$. Then conjugate $a^{t}$ by $a^{i}$ and $a^{i} b$ thus we have

$\left(a^{t}\right)^{a^{i}}=a^{i} a^{t} a^{-i}=a^{t}$.

We now conjugate $a^{t}$ by $a^{i} b$. By using Lemma 2.1 and for $1 \leq t<2^{\alpha}$ we have

$$
\begin{aligned}
\left(a^{t}\right)^{a^{i} b} & =a^{i} b a^{t} a^{-i r} b \\
& =a^{i} b a^{t-i r} b \\
& =a^{i+(t-i r) r} \\
& =a^{i+\left(t-i\left(2^{\alpha-1}-1\right)\right)\left(2^{\alpha-1}-1\right)} \\
& =a^{t 2^{\alpha-1}-t} .
\end{aligned}
$$

If $t$ is even, then $a^{t}$ is a central element and $\left[a^{t}\right]=$ $\left\{a^{t}, a^{-t}\right\}=\left\{a^{t}\right\}$. If $t$ is odd then

$\left[a^{t}\right]=\left\{a^{t}, a^{2^{\alpha-1}-t}\right\}$

Contains $\frac{2^{\alpha}-2}{2}=2^{\alpha-1}-1$ conjugacy classes of order two. Next we conjugate $a^{t} b$ by $a^{i}$ and $a^{i} b$ respectively. Thus for $1 \leq t<2^{\alpha}$

$$
\begin{aligned}
\left(a^{t} b\right)^{a^{i}} & =a^{i} a^{t} b a^{-i} \\
& =a^{i(1-r)+t} b \\
& =a^{2 i-i 2^{\alpha-1}+t} b
\end{aligned}
$$

and

$$
\begin{aligned}
\left(a^{t} b\right)^{a^{i} b} & =a^{i} b a^{t} b b^{-1} a^{-i} \\
& =a^{i} a^{(t-i) r} b \\
& =a^{i+(t-i)\left(2^{\alpha-1}-1\right)} b \\
& =a^{(t-i) 2^{\alpha-1}+2 i-t} b .
\end{aligned}
$$

Therefore, 
$\left[a^{t} b\right]=\left\{a^{2 i-i 2^{\alpha-1}+t} b, a^{(t-i) 2^{\alpha-1}+2 i-t} b \mid 0 \leq i \leq 2^{\alpha}-\right.$

$1\}$

$$
\begin{aligned}
= & \left\{a^{t} b, a^{t 2^{\alpha-1}-t} b, \ldots, a^{2^{\alpha}-2^{2 \alpha-2}+t} b\right. \\
& \left.a^{\left(t-2^{\alpha-1}\right) 2^{\alpha-1}+2^{\alpha}-t} b\right\} .
\end{aligned}
$$

For $t=1$, we have

$[a b]=\left\{a b, a^{2^{\alpha-1}-1} b, \ldots, a^{2^{\alpha}-2^{2 \alpha-2}+1} b\right.$,

$\left.a^{\left(1-2^{\alpha-1}\right) 2^{\alpha-1}+2^{\alpha}-1} b\right\}=\left\{a^{k} b \mid k\right.$ is odd $\}$.

For $t=2$, we have

$\left[a^{2} b\right]=\left\{a^{2} b, a^{2^{\alpha-1}-2} b, \ldots, a^{2^{\alpha}-2^{2 \alpha-2}+1} b\right.$,

$\left.a^{\left(2-2^{\alpha-1}\right) 2^{\alpha-1}+2^{\alpha}-2} b\right\}=\left\{a^{k} b \mid k\right.$ is even $\}$.

Thus, there are two conjugacy classes with $2^{\alpha-1}$ element.

On the other hand, $Z(G)=\left\langle a^{2^{\alpha-1}}, b^{2}\right\rangle=\left\langle a^{2^{\alpha-1}}\right\rangle$ contains $|Z(G)|=2$ conjugacy classes. Therefore we have

$k(G)=2^{\alpha-1}+3$.

Theorem 3.4 Let $\mathrm{G}$ is a non-abelian 2-group and

$G \cong\left\langle a, b \mid a^{2^{\alpha}}=b^{2}=1,[b, a]=a^{2^{\alpha-1}}\right\rangle$,

where $\alpha \geq 2$. Then $k(G)=2^{\alpha}+2^{\alpha-2}$.

Proof. This group is the quasi-dihedral group $Q D_{2^{\alpha+1}}$ of order $\quad 2^{\alpha+1}$. Using Lemma 2.2, $Z(G)=\left\langle a^{2}\right\rangle$ and $|Z(G)|=2^{\alpha-1}$. This group is a split group of class greater than 2. We obtain $k(G)$ by computing the number of $x^{G}$ for $x \in G$. Note that an arbitrary element of $G$ can be written uniquely in the form $G=\left\{a^{i} b^{j} \mid 0 \leq i<2^{\alpha}, 0 \leq j<2\right\}$.

Also, $Z(G)=\left\langle a^{2}, b^{2}\right\rangle=\left\langle a^{2}\right\rangle$. Moreover, from Lemma 2.1 we have

$\left(a^{i} b^{j}\right)^{a^{s} b^{t}}=a^{s} b^{t} a^{i} b^{j} b^{-t} a^{-s}=a^{s\left(1-r^{j}\right)+i r^{t}} b^{j}$,

where $r=2^{\alpha-1}+1$. Since $b^{2}=1$, it is convenient to work with two forms $a^{k}$ and $a^{k} b$. Hence, we can apply again Lemma 2.1 to find the $\left|x^{G}\right|$ for some $x \in G$. Thus

$\left(a^{i}\right)^{a^{s} b}=a^{s} b a^{i} b a^{-s}=a^{s+i r-s r^{2}}=a^{i\left(2^{\alpha-1}+1\right)}$,

because $|a|=2^{\alpha}$. Similarly $\left(a^{i}\right)^{a^{s}}=a^{i}$. Hence

$\left.a^{i}\right]=\left\{a^{i}, a^{i\left(2^{\alpha-1}+1\right)}\right\}$.

If $i$ is even then $a^{i} \in Z(G)$ and $\left.a^{i}\right]$ is the singleton $\left\{a^{i}\right\}$.

If $i$ is odd then

$\left.a^{i}\right]=\left\{a^{i}, a^{2^{\alpha-1}+i}\right\}$.

In this case we have $\frac{2^{\alpha} / 2}{2}=2^{\alpha-2}$ conjugacy classes of

order 2. Likewise, we have

$\left(a^{i} b\right)^{a^{s}}=a^{s(1-r)+i} b=a^{i-s 2^{\alpha-1}} b$

and

$\left(a^{i}\right)^{a^{s} b}=a^{s\left(1-r^{j}\right)+i r^{t}} b^{j}=a^{(s+i)\left(2^{\alpha-1}\right)+i} b$.

Thus

$\left.a^{i} b\right]=\left\{a^{i} b, a^{\left(2^{\alpha-1}+i\right)} b\right\}$.
In this case we have $2^{\alpha-1}$ conjugacy classes with 2 elements. All non-central elements of $G$ are included in one of the classes mentioned above. Also, $Z(G)=$ $\left\langle a^{2}\right\rangle$ contains $|Z(G)|=2^{\alpha-1}$ conjugacy classes. Hence we have

$k(G)=2^{\alpha-2}+2^{\alpha-1}+2^{\alpha-1}=2^{\alpha}+2^{\alpha-2}$

Corollary 1 Let $G$ be a non-abelian 2-group mentioned in Theorem 3.4. Then

$$
P_{r}(G)=\frac{2^{\alpha-1}+2^{\alpha-2}}{2^{\alpha+1}}=\frac{5}{8}
$$

Proof By using Gustafson's formula $P_{r}(G)=\frac{k(G)}{|G|}$,

the result follows.

Corollary 2 Let $G$ be a non-abelian 2-group given in Theorems 3.1-3.3. Then the commutativity degree of $G$ is $P_{r}(G)=\frac{2^{\alpha-1}+3}{2^{\alpha+1}}$.

Proof. Ulsing Eq. (1.1), the result holds.

\section{Conclusion}

The conjugacy classes and commutativity degree of dihedral groups, semi-dihedral groups and generalized quaternion groups are the same.

\section{Acknowledgement}

The first author would like to acknowledge Universiti Teknologi Malaysia, Johor Bahru for his International Doctoral Fellowship (IDF) award.

\section{References}

1. Beuerle JR (2005) An elementary classification of finite metacyclic $p$-groups of class at least three. Algebra Colloq. 12, 553-562.

2. Erdos $P$ and Turan $P$ (1968) On some problems of statistical group theory. Acta Maths. Acad. Sci. Hung. 19, 413-435.

3. Erfanian A and Russo $F$ (2009) Isoclinism in probability of commuting $n$-tuples. Ital. J. Pure Appl. Maths. 25, 27-36.

4. Gustafson WH (1973) What is the probability that two groups elements commute? Am. Maths. Monthly. 80, 1031-1304.

5. Huppert B (1998) Character theory of finite groups: De Gruyter Press, Berlin.

6. Jaikin-Zapirai A (2003) On the number of conjugacy classes in finite $p$-groups. J. Lond. Maths. Soc. 68(2), 699-711.

7. Kanti $R$ and Kumar $A$ (2010) On a lower bound of commutativity degree. Rend. Circ. Mat. Palermo. 59, 137-142.

8. King BW (1973) Presentations of Metacyclic Groups, Bull. Aust. Math. Soc. 8, 103-131.

9. Moradipour K, Sarmin NH and Erfanian A (2012) Conjugacy classes and commutativity degree of metacyclic $p$-groups. ScienceAsia. 38 (1), 113-117.

10.Sherman G (1979) A lower bound for the number of conjugacy classes in a finite nilpotent group. Pacific J. Maths. 80, 253-254.
K.Moradipour et al. Indian J.Sci.Technol. 